Rev. Adm. Saúde - Vol. 18, № 70, jan. - mar. 2018

http://dx.doi.org/10.23973/ras.70.77

RELATO DE CASO

\title{
Sistemas de informação em saúde e sua complexidade
}

Health information systems and their complexity

\section{Olímpio J. Nogueira V. Bittar¹, Márcio Biczyk², Mário Ivo Serinolli³, Márcia C. Zago Novaretti ${ }^{3}$, Mara Michele Nunes de Moura ${ }^{4}$}

1. Professor da Universidade Nove de Julho, assessor de Gabinete da Secretaria de Estado da Saúde de São Paulo

2. Diretor de Tecnologia da Informação da Secretaria de Estado da Saúde de São Paulo.

3. Professor da Universidade Nove de Julho.

4. Aluna do Curso Profissionalizante em Gestão de Serviços de Saúde da Universidade Nove de Julho

\section{RESUMO}

A saúde da população e a prestação de serviços de saúde estão expostos a influências externas e internas que podem comprometer tanto as condições de saúde da população como facilitar ou dificultar a obtenção da qualidade, produtividade ou custos de prover serviços. Especificamente, no que diz respeito a programas e serviços de saúde, as variáveis externas são epidemiológicas, geográficas, demográficas, socioeconômicas, culturais e de mercado, devendo ser conhecidas e trabalhadas, visando efetividade e eficiência. Quanto às internas, se devem a milhares de fatores, como organizacionais, de recursos pessoal e material, financeiros, informacionais, fluxos operacionais internos, necessitando de tecnologia da informação para possibilitar agregação e tomada de decisão. Objetivou-se neste estudo, levantar os sistemas de informação do Sistema Único de Saúde, conhecer internamente as unidades de saúde, para possibilitar o desenho de novos sistemas e facilitar a interoperabilidade dos existentes, que são muitos, 
permitindo a integração dos mesmos e a obtenção de dados, informação e conhecimento para tomada de decisões.

Palavras-chave: administração de serviços de saúde, sistemas de informação, processamento automatizado de dados

\begin{abstract}
Populations health and healthcare services are exposed to internal and external influences that might compromise patient conditions, as well as facilitate or make it difficult to obtain quality, productivity or cost increase. More specifically, concerning services and programs in healthcare, external variables such as epidemiological, geographical, demographic, social, economic, cultural and market related, shall be known and worked out, aiming its effectiveness and efficiency. Regarding internal variables, these are related to a thousand factors like: administrative, organizational, human and material resources, finances, information and operations flows, among others. Currently, all those areas and fields require Information Technology (IT) to make decision making possible. In this study, we aim at analyzing public sector healthcare Information Systems (IS), their use at the entire healthcare process, building a unified and integrated view, as well as to understand how data, information and knowledge are generate, in order to provide useful decision making for healthcare professionals and managers.
\end{abstract}

Keywords: health services administration, health planning, information systems, automatic data processing

\title{
INTRODUÇÃO
}

Os sistemas de informação em saúde (SIS) devem contribuir para a melhoria da qualidade e da produtividade da assistência de saúde, possibilitando a realização de pesquisas e atividades de ensino. A gestão da informação possibilita que os profissionais de saúde desempenhem as atividades com efetividade e eficiência, integrando a informação, facilitando a comunicação, coordenando as ações entre os múltiplos membros das equipes, fornecendo meios para apoio financeiro e administrativo. A eficiência está relacionada à otimização do uso de recursos para a realização dos diversos processos desempenhados pelos profissionais, tanto no cuidado direto, como na administração ${ }^{1,2}$.

O desenvolvimento de SIS visa suprir as necessidades de interação das equipes para a coordenação do cuidado nos atendimentos. É um conjunto de componentes inter-relacionados que coletam, processam, armazenam e 
distribuem informações para a tomada de decisões no âmbito estratégico e operacional, em atenção à diversidade das organizações, dos profissionais e dos processos envolvidos nas operações técnicas e administrativas. Incluem componentes básicos da tecnologia da informação (TI), como técnica, desenvolvimento, uso e o gerenciamento ${ }^{2,3}$.

A estratégia de gestão é fundamental para o bom sucesso de uma instituição, requerendo eficácia operacional, que, depende da interação com os recursos de $\mathrm{TI}^{2,3}$.

Os SIS desenvolvidos no SUS baseiam-se nas necessidades da informação para gestão e monitoramento de situações de risco, para o controle de produtividade e repasse de recursos financeiros. Seguem políticas de saúde, estratégias de gestão e normas administrativas ${ }^{4}$.

Inúmeros SIS são utilizados pelas organizações de saúde, públicas e privadas, adotadas com o intuito de reduzir custos e aumentar a qualidade dos serviços prestados. Desenvolvidos por diferentes fornecedores possuem arquiteturas, bases de dados e infraestruturas divergentes. Com isso, são criados aplicativos incapazes de se comunicarem entre si, gerando problemas de interoperabilidade ${ }^{5}$.

A área da saúde é influenciada por expressivo número de variáveis internas e externas que interferem nos processos saúde-doença e na administração de programas, serviços e unidades de saúde. Há dificuldades de padronização de insumos, métodos, técnicas e processos tanto na infraestrutura como nas áreas fins devido à grande diversidade de categorias profissionais (14 exclusivas de saúde) além de outras especialidades profissionais que se ocupam das subáreas relacionadas.

As diversas formas jurídicas, administração direta, indireta, fundações, associações, entidades privadas filantrópicas, beneficentes e que visam lucro, exigem por força legal múltiplos controles e prestações de contas.

As três esferas de governo, federal, estadual e municipal, tem autonomia de gestão, entretanto, precisam de informações geradas por diferentes órgãos (próprios ou contratados). A articulação, a gestão, a regulação, a avaliação e o financiamento dos sistemas de saúde ficam prejudicados pelas dificuldades de tráfego de informações entre as três esferas.

O Sistema Único de Saúde (SUS) é complementado pelo Sistema Supletivo de Saúde (SS), porém, na prática não possuem conexões informacionais e operam como sistemas de saúde, distintos, cada um com suas plataformas.

A integração entre sistemas de informação é fundamental para a boa gestão de sistemas e serviços de saúde e é definido com o compartilhamento de uma única base de dados e envolvendo todos os níveis de hierarquia organizacional (estratégico, tático e operacional). O ideal seria um banco de dados comum para atender todas as funções das organizações de saúde, incluindo a gestão das operações técnicas e de suporte administrativo e logístico e informações 
sobre os clientes. Custos de projetos de integração são altos e sujeitos a falhas, pois envolvem muitos processos e necessitam de vários testes ${ }^{6,7,8}$.

A utilização de soluções encontradas no mercado tem sido considerada um diferencial uma vez que otimizam operações em termos de velocidade e valor, reduzindo desperdícios, algumas permitem a integração de funções, troca de informações e de fluxo, bem como a integração de funções de setores diversos (contabilidade, finanças, recursos humanos, operações, marketing, logística. No entanto, a implementação ou aquisição de ferramentas comerciais pode significar quantidade substancial de recursos $6,9,10$.

No âmbito hospitalar, ao longo dos anos, surgiram gerações de sistemas de informação que priorizam a integração de dados clínicos e administrativos, com o objetivo de quantificar e qualificar o atendimento, reduzir custos e obter informações relevantes que compõem um perfil da saúde em uma determinada região. O prontuário eletrônico do paciente (PEP) tornou-se a principal ferramenta com a qual médicos e equipes precisam lidar em suas atividades diárias. Entretanto, apesar dos aparentes benefícios trazidos pela adoção do $\mathrm{PEP}$, o avanço dessa tecnologia no setor de saúde exige não só um investimento de alto custo por parte das instituições de saúde, como também uma capacitação dos profissionais de saúde e um estudo destinado a melhorar as práticas de registro ${ }^{10,11}$.

A padronização de processos e disponibilidade de informações provenientes de um único banco de dados facilita o acesso às funções estratégicas e resulta em ganhos expressivos de produtividade, eliminando duplicidades e disponibilizando informações integradas que podem ser utilizadas pelos diversos níveis de comando incluindo a totalidade dos serviços de atenção primária, secundária e terciária, além das estruturas de comando centralizadas municipais, estaduais e federais ${ }^{9}$.

As maneiras de acessar informações vitais que podem ter sido armazenadas em uma série de aplicações diferentes pode influenciar diretamente o sucesso de uma gestão. Isso também se aplica às organizações de saúde, uma vez que a agilidade de acesso às informações é fator crucial para a qualidade $e$ eficiência da prestação do serviço, sendo crítico inclusive em situações em que vidas estão em risco.

Desta forma, a utilização de SIS é o recurso para a gestão estratégica em saúde. É o suporte para a organização administrativa e técnica, a coleta de dados, o armazenamento, o processamento das informações dos pacientes, o auxílio ao diagnóstico, a prescrição dos medicamentos e cuidados adequados a cada situação em que o paciente estiver envolvido ${ }^{1,11,12}$.

Com o objetivo de contribuir para a interoperabilidade dos sistemas de informação no SUS, listados neste estudo, detalham-se as condições internas das unidades de saúde que influenciam nos resultados das operações que merecem ser analisadas e sintetizadas para efetividade do processo de integração. 


\section{METODOLOGIA}

Estudo descritivo, transversal, tendo como objeto os sistemas de informação utilizados por órgãos de planejamento e direção em saúde e as dificuldades de integração dos diversos tipos de sistemas de informação existentes necessários ao apoio à tomada de decisões, assim como, na rotina de trabalho nos diversos níveis e funções organizacionais.

Foram relacionados sistemas adotados pelo Ministério da Saúde, Agencia Nacional de Saúde Suplementar, Governo do Estado, Secretaria de Estado da Saúde de São Paulo (SES/SP) e unidades prestadoras de serviços, da administração direta, autarquias e unidades privadas conveniadas.

As condições internas são passíveis de maior controle pelos administradores e pelos profissionais das unidades de saúde, e necessitam de gestão efetiva.

O meio externo, fornece dados e informações sobre condições de saúde e outras influencias sócio econômicas, que interferem na formulação de programas e serviços e administração de unidades. Uma exposição das dimensões internas e externas inerentes às instituições de saúde, que impactam na administração das unidades, como capacidade de produção, estrutura necessária (área física, recursos materiais, informacionais, tecnológicos, humanos e financeiros), diversidade de operações, fluxos, processos, que resultam na saúde da população estão expostas de forma a favorecer o entendimento da complexidade do tema abordado.

\section{RESULTADOS}

\section{Sistemas de informações do SUS e da SES/SP}

As diferentes necessidades e subdivisões da área de saúde, sem uma visão sistêmica, acabam por utilizar quantidade exagerada de softwares e planilhas, sem interligação, dificultando ou mesmo impedindo a comunicação e a transformação de dados em informações e conhecimento.

Na sequência, o panorama da diversidade de sites, sistemas e aplicativos em uso no Ministério da Saúde, no Governo do Estado e na SES/SP, e em unidades próprias e privadas:

- Sites e sistemas do Ministério da Saúde /DATASUS (cerca de 60 aplicativos):

http://portalsaude.saude.gov.br/

http://datasus.saude.gov.br/

AIH, SIA (consultas e APAC), SIM; SINAM; SINASC; AIH; BPA; eSUS AB; SIS-Mama, SIS-colo, Hiperdia, SIGTAP, SISCAM, SISREG, SIS pré-natal, SI PNI, SIOPS, CNES, etc. 
- Sites e sistemas da Agência Nacional de Saúde Suplementar:

www.ans.gov.br

TISS

CIHA

- Sites e sistemas do Governo do Estado de São Paulo (cerca de 50 aplicativos):

http://www.saopaulo.sp.gov.br/

http://www.planejamento.sp.gov.br/

http://www.prodesp.sp.gov.br/

SIAFEM; SIAFÍSICO; GESTÃO RH / Folha de Pagamento; BEC; etc.

- Sites e sistemas desenvolvidos pela SES/SP (cerca de 50 aplicativos):

http://www.saude.sp.gov.br/

http://intranetses.saude.sp.gov.br/

http://gsnet.saude.sp.gov.br

http://exames.s4.sp.gov.br

http://www.farma.saude.sp.gov.br

www.portalfinanceirodogestor.saude.sp.gov.br

http://www.nih.saude.sp.gov.br/

http://www.gestaohospitalar.saude.sp.gov.br/site/

http://sistema4.saude.sp.gov.br/sahe/

Medicamentos SCODES, MEDEX; S4SP; CTX Transplantes; Sistema CROSS, SIVISA, SANI, etc.

- Sistemas adquiridos no mercado pelas unidades SES/SP: Philips Tasy; MV Gestão Hospitalar; Wareline; Totvs Gestão; Gesthos; etc.

- Sistemas das unidades/setores SES/SP, incluindo Data Base System próprios: sistemas internos GIS/CIC; bancos de dados das coordenadorias; planilhas Excel; etc. A convivência de sistemas e planilhas Excel para tomadas de decisão são reais, levando a trabalho e 
retrabalho informações administrativas incompletas, com perda da confiabilidade necessária ao sistema de saúde.

Estes sistemas foram desenvolvidos ou adquiridos à medida que a necessidade de informações para resolver problemas ocorria, sem a preocupação de interligação; na sua maioria as informações não possibilitam, com agilidade, a tomada de decisões administrativas, técnicas, epidemiológicas, ambientais, adequadas à situações de rotina, de urgência e emergências, para ações individuais ou coletivas.

A não integração dos sistemas faz com que haja a necessidade de coletar os dados em diferentes bases e processa-los em outro ambiente, tornando as informações menos confiáveis, acrescendo custo aos orçamentos já deficitários.

A Troca de Informações na Saúde Suplementar - TISS foi estabelecida como um padrão obrigatório para as trocas eletrônicas de dados de atenção à saúde dos beneficiários de planos, entre os agentes da SS. O objetivo foi padronizar as ações administrativas, subsidiar as ações de avaliação e acompanhamento econômico, financeiro e assistencial das operadoras de planos privados de assistência à saúde e compor o Registro Eletrônico de Saúde. A TUSS (Terminologia Unificada do Sistema Suplementar) é baseada na Classificação Brasileira Hierarquizada de Procedimentos Médicos (CBHPM) ${ }^{13}$.

Além deste sistema, na SS, há a coleta de informações por meio da Comunicação de Informação Hospitalar e Ambulatorial (CIHA), permitindo entre outras vantagens o conhecimento de dados administrativos, demográficos e perfil epidemiológico dos pacientes portadores de planos de saúde, tanto de pacientes internados como dos ambulatoriais. A SES/SP já utilizou instrumento semelhante, o Boletim $\mathrm{CAH} 106$, desativado há tempos, utilizados tanto para coleta de dados de pacientes do SUS como da SS, internados nas unidades hospitalares públicas e privadas do Estado ${ }^{14}$.

A Secretaria de Estado da Saúde, por sua vez, com função de coordenação da rede estadual e gestão de unidades próprias, utiliza-se de sistemas desenvolvidos internamente para suas necessidades operacionais, além de outros adquiridos no mercado.

No caso dos hospitais da SES/SP, existem três sistemas distintos, o Núcleo de Informação Hospitalar (NIH) para hospitais da administração direta, o Sistema Gestão Hospitalar para os hospitais vinculados a organizações sociais de saúde e o Sistema para Avaliação de Hospitais de Ensino (SAHE). O estudo destes hospitais de ensino é complementado com informações do DATASUS, principalmente para avaliações da qualidade da assistência prestada, usando inclusive, protocolos da Agency for Healthcare Research Quality (AHRQ). Pesquisas realizadas com estes sistemas podem ser encontradas no site do SAHE, atendendo as necessidades de informação da unidade e do nível central da SES/SP.

Com poder de controle menor as Influências Externas espelham condições demográficas, geográficas, ambientais, epidemiológicas, educacionais, 
culturais, psicossociais, legais, políticas sociais, econômicas, tecnológicas, mercado de saúde (entidades públicas, privadas lucrativas e sem fins lucrativos, planos de saúde), e suas influências nos aspectos de saúde e provisão de programas e serviços.

As informações sobre influências externas estão disponíveis nas mais variadas fontes: sites de Ministérios, Secretarias Estaduais e Municipais de Saúde, Agência Nacional de Saúde Supletiva, Fundação Instituto Brasileiro de Geografia e Estatística - IBGE, Fundação Estadual de Análise de Dados SEADE, Fundação Getúlio Vargas - FGV, Fundação Instituto de Pesquisas Econômicas - FIPE, Confederação Nacional da Indústria - CNI, institutos de pesquisa, associações de classe e de especialidades, conselhos, imprensa, entre outros órgãos que trabalham no sentido de consolidar dados e informações, agregando informações, possibilitando criar parâmetros para suporte ao monitoramento e à decisão ${ }^{15}$.

De outra forma as Influências Internas oferecem maior controle por parte dos gestores podendo ser compreendidas como complexas em decorrência de processos de produção, que ocorrem na área física (consultórios, unidades de internação, laboratórios, tratamento intensivo, unidade cirúrgica), que é gerida por uma estrutura administrativa (áreas e subáreas) que por sua vez é baseada em recursos, prioritariamente humanos, por meio de processos interligados por inúmeros fluxos. Os diversos processos administrativos são registrados em instrumentos administrativos e principalmente do prontuário do paciente. Cada área e subárea tem sua especificidade em termos informacionais, assim como indicadores de saúde e de negócios que permitem medições e acompanhamento servindo como ferramenta para tomada de decisão.

\section{Estrutura do meio interno das instituições de saúde}

No desenvolvimento de sistemas de informação/informatização importa conhecer a complexidade das estruturas administrativas que integram, de forma geral, os sistemas e serviços de saúde, necessárias para o alcance de metas quantitativas e qualitativas. Nas unidades de saúde, cada área é desdobrada em subáreas com cultura própria, que exige técnicas diferenciadas, pessoal especializado, insumos específicos, indicadores e medidas próprias para suas atividades, tais como:

- Conselhos: (administrativo e fiscal) incluem negociações internas e externas, importantes na obtenção e utilização de recursos e na imagem institucional. Entre as atividades destes conselhos estão o conhecimento da situação financeira da empresa, os riscos econômicos, legais, de governança e sustentabilidade, as dinâmicas do setor em que ela atua, criando estratégias para sobrevivência empresarial;

- Infraestrutura: são subáreas que não mantém contato direto com o paciente, suporte para o funcionamento das áreas fim. Exigem informatização, automação, mecanização, racionalização de pessoal e 
otimização de custos e sua composição é: administração, auditoria, biblioteca, centro de esterilização de materiais, creche, farmácia, finanças (orçamento, contabilidade geral, tesouraria, contas a pagar e a receber, contabilidade de custos), informática, jurídica, lavanderia/costura, manutenção (predial, elétrica, eletrônica, hidráulica, equipamentos, marcenaria), marketing (convênios, atendimento ao cliente, divulgação), material (compras, almoxarifado, patrimônio e importação), nutrição e dietética (produção, lactário), ouvidoria, recursos humanos (recrutamento, seleção, treinamento, administração de pessoal e benefícios, engenharia/segurança e medicina do trabalho), serviço de prontuário do paciente (matricula, agendamento internação, arquivo, estatística e faturamento), reprografia, zeladoria (protocolo, vigilância, telefonia, recepção, portaria, estacionamento, elevadores, vestiários, limpeza, transporte e velório);

- Ambulatório: fundamental para a orientação e o acompanhamento de pacientes, monitoramento dos portadores de doenças crônicas e redução do tempo de internação. Com investimento tecnológico podem ter maior resolubilidade, humanização do atendimento e redução de custos e riscos. Tal como a área de internação, deve estar integrado com o restante da rede de saúde de atenção primária, por meio de regulação eficiente, reduzindo filas e demanda reprimida na instituição e facilitando o acesso dos pacientes que necessitem de atenção mais complexa;

- Emergência: atendimento de casos graves. Juntamente com o ambulatório é a porta de entrada do sistema;

- Área complementar de diagnóstico e terapêutica: são verdadeiras linhas de montagem, usadas tanto para diagnóstico como para terapêutica, importantes no aumento da resolubilidade de programas e serviços hospitalares, prevenindo e reduzindo internações. São eles: análises clínicas, anatomia patológica, angiografia/hemodinâmica, ecocardiografia, endoscopia, eletrocardiografia, eletroencefalografia, ergometria, fisioterapia, hemodiálise, holter, litotripsia, mapa, medicina nuclear, potencial evocado, provas em pneumologia, radiologia convencional, radioterapia, ressonância magnética, tomografia, ultrassonografia. Alguns já são feitos à distância, por telemetria. Sistemas automatizados de laboratório e de Radiologia Digital estão se tornando cada vez mais presentes, tanto nos hospitais privados como públicos;

- Internação clínico/cirúrgica: devem ser preservadas para tratamentos agudos e de média e alta complexidade, pois o consumo de recursos é intenso (mais custosas) e, além disso, as internações trazem mais riscos para o paciente. Unidades de internação clínico-cirúrgica, terapia intensiva adulto, pediátrica, neonatal, e seus respectivos elementos;

- Comissões obrigatórias: prontuário do paciente, infecção hospitalar, análise de óbitos e biópsias, ética, ética em pesquisa, farmácia e terapêutica, intra-hospitalar de transplante, nutrição enteral e parenteral, 
brigada de incêndio, comissão interna de prevenção de acidentes, gerenciamento de resíduos, transfusional, apropriação de custos, entretanto, outras (como qualidade, incluindo humanização) podem ser criadas visando à melhoria da qualidade do atendimento aos pacientes, do desempenho e da integração das diversas áreas e subáreas. Sem elas, a interação continua entre as áreas torna-se impossível. Para interações pontuais, lançam mão das estruturas matriciais;

- Pesquisa: uma das grandes dificuldades no sistema é o registro de pesquisas em andamento nas unidades de saúde, bem como o monitoramento necessário para avaliação da representatividade para a comunidade. Pode representar custos elevados; necessita técnicas específicas de administração e negociação com pesquisadores, agências de fomento, indústria e fornecedores. Sistemas de informações completos permitem separar recursos utilizados em pesquisa, evitando que a assistência financie a mesma, que tanto hospital como pesquisador e auxiliares sejam remunerados adequadamente e honestamente. Pesquisas são realizadas na maioria dos 51 hospitais de ensino do Estado, certificados pelos Ministérios da Educação e da Saúde;

- Ensino: repercute na produtividade e nos custos da assistência das unidades formadoras uma vez que em geral o tempo de permanência dos pacientes é maior, idem ao número de exames e procedimentos complementares. Seu monitoramento exige atualização e investimento constante em pessoal especializado, técnicas e conexão entre educador, prestador e gestor. O financiamento do ensino deve ser acompanhado com técnica permitindo a busca de recursos específicos para este fim. Na SES/SP a residência médica consome centenas de milhares de reais, anualmente, formando especialistas em 53 programas. A SES/SP é responsável por apoiar 6.500 bolsas para residência médica e 1.300 para aprimoramento profissional;

- Áreas extra unidade de saúde: inclui tudo que é realizado pela equipe de saúde fora da unidade, como o atendimento e a assistência domiciliar, exemplificando com o programa Estratégia Saúde da Família. Há unidades de saúde com indústria de equipamentos, instrumental, órteses e próteses, farmácia de manipulação.

No caso da SES/SP possui fábrica de medicamentos e vacinas, respectivamente a Fundação para o Remédio Popular - FURP e o Instituto Butantã, cuja informatização deve obedecer a requisitos industriais e de entidades de pesquisa.

De modo prático, a decomposição das grandes áreas em inúmeras subáreas evidencia as particularidades de cada uma, bem como competências específicas que têm que ser desenvolvidas com informações e conhecimento para a execução de operações e obtenção de resultados cuja qualidade tenha impacto positivo na saúde da população e na gestão da unidade. 


\section{Utilização de recursos/insumos - condição interna}

- Humanos (próprios e terceiros) São 14 categorias profissionais de saúde que se desdobram em 346 especialidades, além de outras categorias profissionais de infraestrutura, cuja produção tem particularidades qualitativas e quantitativas. Geralmente, são utilizados sistemas de informações próprios para a área de recursos humanos, que permitam manter sigilo das informações dos funcionários. Entretanto estes sistemas devem permitir ao sistema maior da unidade a importação de dados para cálculos de produtividade de pessoal. Em relação a profissionais terceirizados, o Caderno Técnico de Serviços Terceirizados - CADTERC, do Governo do Estado de São Paulo, trás parâmetros para contratação, por exemplo, o número de funcionários da limpeza por metro quadrado de área, o que auxilia no dimensionamento de funcionários ${ }^{16}$. Algumas categorias profissionais de saúde ainda oferecem resistência à implantação de $\mathrm{TI}$ em unidades de saúde, o que dificulta o pleno aproveitamento dos sistemas e a qualidade das informações.

- Materiais permanentes (edificações, equipamentos, mobiliário, instrumentais, utensílios, veículos). Investimento, manutenção, inventário, depreciação devem ser contabilizados para o bom andamento da produção, financiamento e controle das unidades de saúde.

- Materiais de consumo (insumos médicos e de enfermagem, medicamentos, órteses e próteses, gases medicinais, limpeza, combustíveis, tecidos, escritório, informática, impressos, gêneros alimentícios). Listas dos itens de medicamentos são encontradas no Brasindice (Guia Farmacêutico Brasileiro, 2017) ${ }^{17}$, e na Bolsa Eletrônica de Compras - BEC ${ }^{16}$. A logística de distribuição de medicamentos/materiais (dezenas de milhares de itens) deve manter registros rigorosos (apuração de custos por procedimentos). Controle de estoque, e tomada de preços para aquisição são tarefas corriqueiras que exigem bons sistemas informatizados que permitam transparência, controle do desperdício e principalmente rastreabilidade.

- Utilidade pública (energia: elétrica, solar, gás, eólica, outras; água e esgoto; comunicação - telefone, internet, intranet, correio, transporte). As dificuldades de rateio destes itens se dão pela não disponibilidade de medidores de consumo individual por áreas e subáreas.

- Tecnologia da informação. Informações epidemiológicas, produção, produtividade, qualidade, custo, recursos humanos, materiais, utilidade pública, ambientais, são coletadas, tratadas e disponibilizadas aos administradores das unidades, órgãos centrais, instituições do mercado e governamentais.

- Financeiros. Inúmeras são as formas de custeio e financiamento em saúde, pública ou privada, em todos os níveis, planos de saúde, venda de bens e serviços, pesquisa, ensino, doações. Internamente as 
unidades são detentoras de todos os tipos de custos, gastos e despesas, que necessitam contabilização e rateio entre as diversas áreas e subáreas. Não podem ser desprezados aspectos recentes relativos à judicialização da saúde que, sujeitos à intempestividade das ações ou levam a gastos não planejados, causando distorções orçamentárias, com consequências graves para a gestão de operações das unidades. O registro dos valores físicos e financeiros permite controle, monitoramento, auditoria e fiscalização da movimentação financeira. A estrutura administrativa, contemplando áreas e subáreas das unidades, resultante do regulamento institucional permite que a utilização dos recursos se faça dentro do método da administração: planejamento, organização, direção (coordenação, regulação e negociação) e avaliação (controle, fiscalização, monitoramento, auditoria) complementadas com a utilização de ferramentas de gestão, o que é possibilitado por um efetivo sistema de informação/informatização. O processamento de informações externas e internas é a base para o bom sucesso das operações de saúde, requerendo suporte, desenvolvimento contínuo e capacitação profissional.

- Produtos (consultas, exames, cirurgias, procedimentos invasivos). Indiscutivelmente, unidades de saúde são complexas, complicadas, de alto risco e alto custo, comprovado pelas influencias externas a que são submetidas, nas áreas e subáreas da sua estrutura administrativa, na discriminação dos recursos que são utilizados como insumos para obtenção dos seus produtos: prevenção da doença, promoção da saúde, diagnóstico, tratamento e reabilitação.

A Classificação Internacional de Doenças - CID $10^{18}$ apresenta 2.036 diagnósticos (no caso dos itens com três dígitos e 12.423, no de quatro itens), o que por si só demonstra complexidade. Trabalhar com número elevado de diagnósticos exige ferramentas como o DRG - Diagnostic Related Groups, que possibilitam simplificação e facilidade de operacionalização ${ }^{19}$.

A Tabela de Procedimentos Unificada SUS ${ }^{20}$, ou seja, uma das listas de produtos que unidades de saúde podem produzir, a partir dos diagnósticos do CID 10, conta com algo em torno de 4.557 itens (113 na atenção básica; 1.515 na alta complexidade; 2.289 na média complexidade; e 640 não se aplica complexidade - próteses, diárias, ajuda de custo, cadastro). Um laboratório de análises clínicas pode realizar 3.000 tipos de exames diferentes, (bioquímica, microbiologia, hematologia, virologia, imunologia).

Os procedimentos previstos são realizados por meio de operações exigindo inúmeros processos técnicos e administrativos (protocolos e procedimentos operacionais padrões), nas áreas de infraestrutura, ambulatório, emergência, área complementar de diagnóstico e terapêutica e internação clínico-cirúrgica, acarretando grandes volumes de dados e informações.

Estes produtos podem ser obtidos por operações comandadas a distância, utilizando-se das telessaúde (eletrocardiografia, oftalmologia, diagnóstico por imagem, dermatologia, segunda opinião), permitindo inclusive monitoramento de doenças crônicas, ações de vigilâncias (sanitária e epidemiológica) 
implementação e avaliação de programas e serviços, unindo unidades de saúde, respondendo com rapidez que as situações clínicas e operacionais exigem.

A avaliação destes produtos, exigida por grande quantidade de indicadores qualitativos e quantitativos, só é possível com SIS, banco de dados desenhados e com capacidades específicas para as situações de saúde.

Fluxos/rastreabilidade. Os fluxos existentes em unidades de saúde necessitam de coordenação que permitam ligação entre as operações de produção de serviços e bens de saúde. Podem ser classificados de acordo com suas características e tem logística própria no processo produtivo:

- Pessoas: clientes-pacientes (externos, internos, em emergência, urgência ou rotina), acompanhantes, visitantes, estudantes (médicos residentes, aprimorandos, estagiários), empregados, funcionários de empresas terceirizadas, representantes de laboratório;

- Insumos e materiais: materiais permanentes (equipamentos, instrumentais, móveis), materiais de consumo, medicamentos, drogas, isótopos radioativos, contrastes, gêneros alimentícios, órteses e próteses, roupas e outros itens de hotelaria, estoques;

- Exames: amostras laboratoriais, peças anatômicas, resultados de exames;

- Informações: prontuário do paciente, agendamento de consultas, cirurgias, exames, procedimentos invasivos e de outros tipos, laudosatestados-guias, contas de convênios, faturamento SUS, boletins de informações, saídas de pacientes - altas e óbitos, transferências internas e externas, listas de espera, controle de leitos, listas de checagem, agendamentos, notícias para mídia, protocolos, informações técnicas e administrativas, informações externas;

- Instrumentos contratuais: convênios, contratos de gestão, protocolos de cooperação, contratos de manutenção e calibração de equipamentos, protocolos de pesquisa, certificações, homologações, explicitam produtos e contrapartidas, gerando documentos que contém dados e informações;

- Resíduos: subdivididos em: lixo orgânico (infectante), lixo inorgânico (comum), lixo radioativo, lixo químico, perfuro cortante, descartáveis, que podem ser submetidos a coleta seletiva e reciclagem, devendo ser catalogado e cadastrado, observada a legislação.

A validação destes fluxos, por meio de procedimentos operacionais padrão, se obriga a responsabilização, o que deve ser feito por meio da certificação digital, mecanismo tecnológico utilizado para identificar e autenticar usuários, senhas, sites e sistemas eletrônicos, contendo conjunto de informações codificadas e criptografadas referentes à entidade para a qual o certificado foi emitido, seja 
uma empresa, uma pessoa física ou computador, redundando em segurança e agilidade das ações.

\section{CONSIDERAÇÕES FINAIS}

Informação consistente e de qualidade é ferramenta fundamental para tomada de decisão, para a ampliação de possibilidades de racionalização de ações, coibição do desperdício, controle e alocação correta de recursos. Informação significa financiamento ${ }^{20}$.

Na cultura interna das unidades de saúde, predomina a visão de curtíssimo prazo, em detrimento ao médio e longo prazo, fazendo com que investimentos em equipamentos e insumos tenham prevalência sobre a estratégia de TI que em curto prazo pode significar investimentos e custos elevados, entretanto não há mais como adiar investimentos maciços em tecnologias de informação e gestão e planejamento para equiparar a área da saúde a outras áreas de negócios que desenvolveram acompanhando os avanços em ciência e tecnologia.

A administração da economia de recursos não deve significar sempre o baixo investimento e sim os bons investimentos que em longo prazo terão seus valores amortecidos devido à eficiência alcançada e diminuição de desperdício e retrabalho. Mais recursos para aperfeiçoamento de equipes e maior transparência nos processos.

Estratégias bem definidas de TI na gestão de saúde demandam inúmeros recursos e exigem desenho claro quanto ao tipo de informação, fluxo e acesso a banco de dados, envolvendo processos em diferentes níveis, profissões, áreas e subáreas de uma mesma instituição.

Em tempos de inteligência artificial, computação cognitiva e machine learning, além do debate sobre aspectos de governança corporativa, sustentabilidade (ambiental, social e econômica) e aplicação de regras de compliance, permitindo evitar ou corrigir erros, desperdícios, abusos e fraudes, é imperioso a utilização de sistemas de informações robustos, confiáveis e integrados.

A partir da análise sistêmica será possível observar as técnicas mais apropriadas para o planejamento de integração e seu grau de complexidade nos processos. A integração de sistemas de informação pode ser realizada de várias formas de acordo com as necessidades definidas na estratégia institucional.

O importante é os planejadores envolvidos se preocupem em criar um ambiente propício à resiliência entre as áreas envolvidas de modo a viabilizar a integração esperada e melhorar a qualidade da informação no âmbito da instituição. 


\section{REFERÊNCIAS}

1. Marin HF. Sistemas de informação em saúde: considerações gerais. $J$ Health Inform 2010; 2(1): 20-24.

2. Brasil. Ministério da Saúde, Estratégia e-Saude para o Brasil, Comitê Gestor da Estratégia e-Saúde, Brasília, 2017. [acessado 2017, set 09]. Disponível em:

http://portalarquivos.saude.gov.br/images/pdf/2017/julho/12/Estrategiae-saude-para-o-Brasil.pdf

3. Pires MRGM, Gottems LBD, Vascocelos Filho JE, Silva KL, Gamarski R. Sistema de informação para a Gestão do Cuidado na Rede de Atenção Domiciliar (SI GESCAD): subsídio à coordenação e à continuidade assistencial no SUS. Cien -Saúde Colet 2015; 20(6):1805-1814.

4. Gava M. Ferreira LS, Palhares D, Mota ELA. Incorporação da tecnologia na Atenção Básica do SUS no Nordeste do Brasil: expectativas e experiências Cien Saude Colet 2016; 21(3):891-902.

5. Siqueira OMP, Oliveira RAN, Oliveira AA. Integração de sistemas de informação em saúde com a utilização de Service Oriented Architecture (SOA) Revista de Gestão da Tecnologia e Sistemas de Informação 2016;13(2):255-274.

6. Dezdar S, Ainin S. The influence of organizational factors on successful ERP implementation. Manag Decision 2011;49(6):911-926.

7. Katerattanakul P, Lee JJ, Hong S. Effect of business characteristics and ERP implementation on business outcomes: an exploratory study of Korean manufacturing firms. Management Research Review 2014;37(2),186-206. [acessado 2017 set 09] Disponível em: https://doi.org/10.1108/MRR-102012-0218

8. Ribeiro, N. T., Serinolli, M. I., Novaretti, M. C. Z., \& Souza, I. (2016). A análise de adesão de competências organizacionais relacionadas à pósimplementação de sistemas ERP em hospitais públicos da administração direta de São Paulo - SP. Espacio. 37(19),3 [acessado 2017 set 09] Disponível em: http://www.revistaespacios.com/a16v37n19/16371903.html

9. Bonaparte I, Austin N, Okoro E. Strategic decision making at enterprise resource planning: chief financial officer at the crossroads. Journal of Business Case Studies 2015;11(1):41-48. [cited 2016 Oct $7^{\text {th }}$. Available from:

https://www.cluteinstitute.com/ojs/index.php/JBCS/article/view/9053/9 $\underline{054}$

10. Rondina JM, Canêo PK, Campos MS. Conhecendo a experiência de implantação do prontuário eletrônico do paciente no hospital de base de 
São José do Rio Preto. Rev. Adm. Hospitalar e Inovação em Saúde. v. 13, n. 1 (2016): pag. 43-52

11. Cavalcante RP, Pinheiro MM. Política nacional de informação e informática em saúde: avanços e limites atuais Perspectivas em Gestão \& Conhecimento, João Pessoa 2011;1(2):106-119.

12. Gutierrez MA. Sistemas de informação hospitalares: progressos e avanços Journal of Health Informatics 2011; 3(2):I-II.

13. Associação Médica Brasileira. Classificação brasileira hierarquizada de procedimentos médicos. [acessado 2017 set 18]. Disponível em: https://amb.org.br/_arquivos/_downloads/CBHPM-2016.pdf

14. São Paulo (Estado) - Secretaria da Saúde. Portaria CAH nº 242 de 5 de setembro, de 1984. Dispõe sobre encaminhamento do Boletim Modelo CAH 106. Diário Oficial, do Estado de 11 de setembro de 1984. Seção 1, p. 13.

15. Bittar OJNV, Mendes JDV, Magalhães A. Saúde e influências externas. Boletim Epidemiológico Paulista - BEPA 2017;14(161):27-55. [acessado 2017 ago 29]. Disponível em:

www.saude.sp.gov.br/.../ses/.../bepa_161_saude_e_influencias_externas_g ais_21.pdf

16.São Paulo (Estado) Secretaria da Fazenda. Estudos Técnicos de Serviços Terceirizados - Cadterc. [acessado 2017 set 9]. Disponível em:

http://www.cadterc.sp.gov.br

17. Guia Farmacêutico Brasindice: [acessado 2017 ago 13]. Disponível em: http://www.brasindice.com.br/

18. Classificação Estatística Internacional de Doenças e Problemas Relacionados à Saúde CID-10. [acessado 2017 jun 20]. Disponível em: www.datasus.gov.br/cid10/V2008/cid10.htm/

19. Couto, R C, Pedrosa, T M G, Rosa M B, Erros acontecem. A força da transparência para o enfrentamento dos eventos adversos assistenciais em pacientes hospitalizados. Construindo um sistema de saúde mais seguro. IESS-Instituto de Estudos da Saúde Suplementar, Belo Horizonte, 2016. 48 pag.

20. Brasil. Ministério da Saúde. Tabela Unificada de Procedimentos. [acessado 2017 set 15]. Disponível em: http://sigtap.datasus.gov.br/tabelaunificada/app/sec/inicio.jsp 
Recebido: 10 de janeiro de 2018. Publicado: 12 de janeiro de 2018

Correspondência: Olímpio Bittar. E-mail: olimpiobittar@gmail.com

Conflito de Interesses: os autores declararam não haver conflito de interesses.

(C) This is an Open Access article distributed under the terms of the Creative Commons Attribution License, which permits unrestricted use, distribution, and reproduction in any medium, provided the original work is properly cited 
